\title{
Brain Activity during Motivative Exercise Versus Passive ROM Exercise by fMRI
}

\author{
Yoshiko Morita ${ }^{1}$, Shigeo Takizawa ${ }^{2}$ \\ ${ }^{1}$ Okayama rehabilitation hospital ${ }^{2}$ Biophilia Institute, Designated by MEXT
}

\begin{abstract}
In the chronic stages of aging or illness, less staffs are employed in both medical and care faculties in Japan than acute stages. This situation might have the serious risk of many patients returning to bedridden states easily. To solve this problem, we have recognized "The Motivative Exercise" as being very useful. In Motivative Exercise, we use simple tools instead of therapists' hands to have them exercise their legs by themselves. We could prove the effectiveness of this rehabilitation method by f MRI to observe brain activities.
\end{abstract}

Keywords: Motivative exercise, f MRI, Chronic stage of aging, Rehabilitation

\section{Introduction}

First, we would like to explain how the Japanese medical insurance system works. The main concept of the Japanese public medical system is to distribute equal services among all people. In response to increasing seniors' populations, the Japanese Care Insurance System was established in April 2000 to lessen the burden of expanding medical expenses. Since then, Medical and Care Insurance have cooperated with each other according to the recovery stages. Medical insurance carries weight mostly in acute phase and medical expenses refocused to acute phase within limited days, usually around two weeks, by following the American DPC system ${ }^{1)}$. Following the acute phase, some patients need rehabilitation services to come back home again. Furthermore in the chronic phase, the rehabilitation services are provided not from Medical Insurance but by Care Insurance.

On the other hand, in medical hospitals, the numbers of employees are decreasing from acute towards chronic faculties just like in the Care Insurances' nursing homes. Against these economic situations, many patients tend to expect the restoration from disabilities through. Especially in outpatients' clinic, we have been urged to decrease medical rehabilitation services guided by national fiscal policy and they have suffered from the dilemma.

To solve this problem in the chronic stage, we have noticed the Motivative Exercise very useful and helpful in restoring the independent life ${ }^{2), 3), 4)}$. The method of rehabilitation with the Motivative Exercise was patented in the United States of America by the author ${ }^{5)}$. We are sure to prove the effectiveness of the Motivative Exercise by observation of brain activities by $f$ MRI.

Received 10 September, 2012 


\section{Materials and Methods}

The Ethics Committee of the Okayama Rehabilitation hospital permitted this study, and the registered clinical trial number is UMIN000006559.

\subsection{Subjects}

Subjects are chronic stroke patients. All patients attend a day care program belonging to my hospital, using Care Insurances Day Care Services. 13 patients: consisting of 4 female, 9 male. Ages: 54 to 83 and average is $67 \mathrm{y}-0$. Locomotion ability; all are house-ambulatory, and some of them community walkers. The duration from the onset of disease is from 5 months to 11years, the average is around 5years.

\subsection{Task}

The task is an ankle joints' exercise. The Motivative Exercise to both ankle joints versus passive ROM exercises to a paralyzed ankle joint by physical therapists' hands. Passive ROM to the affected side only, but in Motivative Exercise, patients can exercise using Pata on the non-affected to the affected side or simultaneously to both side exercises.

Table 1. Subjects and their disease, impairments and disabilities

\begin{tabular}{|c|c|c|c|c|c|c|}
\hline & Age & Sex & disease & duration & impairment & ambulatory \\
\hline 1 & 53 & $\mathrm{M}$ & CVA & $5 \mathrm{~m}$ & $\begin{array}{l}\text { Right Hemiplegia } \\
\text { BRS U/E2 L/E3 }\end{array}$ & $\begin{array}{l}(\mathrm{P}) \text { in side } \\
(\mathrm{P}) \text { outside }\end{array}$ \\
\hline 2 & 62 & $\mathrm{M}$ & CVA & $6 y 7 m$ & $\begin{array}{l}\text { Left Hemiplegia } \\
\text { BRS U/E2 L/E3 }\end{array}$ & $\begin{array}{l}(\mathrm{P}) \text { in side } \\
(\mathrm{N}) \text { outside }\end{array}$ \\
\hline 3 & 64 & $\mathrm{M}$ & CVA & $4 y 10 m$ & $\begin{array}{l}\text { Left Hemiplegia } \\
\text { BRS U/E2 L/E4 }\end{array}$ & $\begin{array}{l}(\mathrm{P}) \text { in side } \\
(\mathrm{N}) \text { outside }\end{array}$ \\
\hline 4 & 78 & $\mathrm{~F}$ & CVA & $1 \mathrm{y} 8 \mathrm{~m}$ & $\begin{array}{l}\text { Left Hemiplegia } \\
\text { BRS U/E2 L/E3 }\end{array}$ & $\begin{array}{l}(\mathrm{P}) \text { in side } \\
(\mathrm{N}) \text { outside }\end{array}$ \\
\hline 5 & 65 & $\mathrm{M}$ & CVA & $4 y 10 \mathrm{~m}$ & $\begin{array}{l}\text { Left Hemiplegia } \\
\text { BRS U/E2 L/E2 }\end{array}$ & $\begin{array}{l}(\mathrm{P}) \text { in side } \\
(\mathrm{N}) \text { outside }\end{array}$ \\
\hline 6 & 60 & $\mathrm{M}$ & CVA & $10 \mathrm{y} 10 \mathrm{~m}$ & $\begin{array}{l}\text { Left Hemiplegia } \\
\text { BRS U/E2 L/E2 }\end{array}$ & $\begin{array}{l}(\mathrm{P}) \text { in side } \\
\text { outside WC }\end{array}$ \\
\hline 7 & 67 & $\mathrm{M}$ & CVA & $3 y 10 m$ & $\begin{array}{l}\text { Left Hemiplegia } \\
\text { BRS U/E2 L/E2 }\end{array}$ & $\begin{array}{l}(\mathrm{P}) \text { in side } \\
\text { outside WC }\end{array}$ \\
\hline 8 & 75 & F & CVA & $2 y$ & $\begin{array}{l}\text { Right Hemiplegia } \\
\text { BRS U/E5 L/E5 }\end{array}$ & $\begin{array}{l}(\mathrm{P}) \text { in side } \\
(\mathrm{N}) \text { outside }\end{array}$ \\
\hline 9 & 71 & $\mathrm{M}$ & CVA & 6y $11 \mathrm{~m}$ & $\begin{array}{l}\text { Left Hemiplegia } \\
\text { BRS U/E2 L/E3 }\end{array}$ & $\begin{array}{l}(\mathrm{P}) \text { in side } \\
(\mathrm{N}) \text { outside }\end{array}$ \\
\hline 10 & 83 & $\mathrm{~F}$ & CVA & $6 y 1 \mathrm{~m}$ & $\begin{array}{l}\text { Left Hemiplegia } \\
\text { BRS U/E3 L/E4 }\end{array}$ & $\begin{array}{l}(\mathrm{P}) \text { in side } \\
\text { outside WC }\end{array}$ \\
\hline 11 & 69 & $\mathrm{M}$ & CVA & $11 \mathrm{y} 7 \mathrm{~m}$ & $\begin{array}{l}\text { Left Hemiplegia } \\
\text { BRS U/E4 L/E4 }\end{array}$ & $\begin{array}{l}(\mathrm{P}) \text { in side } \\
(\mathrm{N}) \text { outside }\end{array}$ \\
\hline 12 & 72 & $\mathrm{~F}$ & CVA & $3 y 9 m$ & $\begin{array}{l}\text { Left Hemiplegia } \\
\text { BRS U/E5 L/E5 }\end{array}$ & $\begin{array}{l}\text { (P)in side } \\
(\mathrm{N}) \text { outside }\end{array}$ \\
\hline 13 & 59 & $\mathrm{M}$ & CVA & $1 \mathrm{y} 5 \mathrm{~m}$ & $\begin{array}{l}\text { Left Hemiplegia } \\
\text { BRS U/E2 L/E3 }\end{array}$ & $\begin{array}{l}(\mathrm{P}) \text { in side } \\
(\mathrm{N}) \text { outside }\end{array}$ \\
\hline
\end{tabular}




\subsection{Protocol}

The protocol is that the trial consists of $45 \mathrm{~s}$ rest - 30s task - 30s rest. Three trials are analyzed automatically by f MRI Company SIGNA ECITE 1.0T. The Pata devises with rubber used in these studies to expose to MRI. Pata for stroke and hemiplegic patients, the non-paralytic limb can move the other paralytic limb by working with this device simultaneously. Patients can do both ankle exercises easily with these simple tools while in a sitting wheel chair position. In fMRI we can't use metal materials. (Fig. 1A) Professor Tanaka, Keio University and his students have repaired the Pata using metal-less rubber instead of a steel spring. (Fig. 1B)

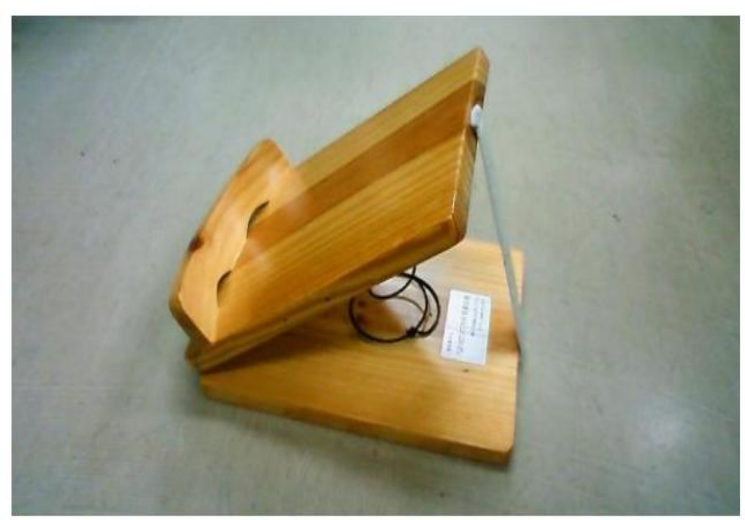

Fig. 1A. Pata ( clinical type with steel spring)

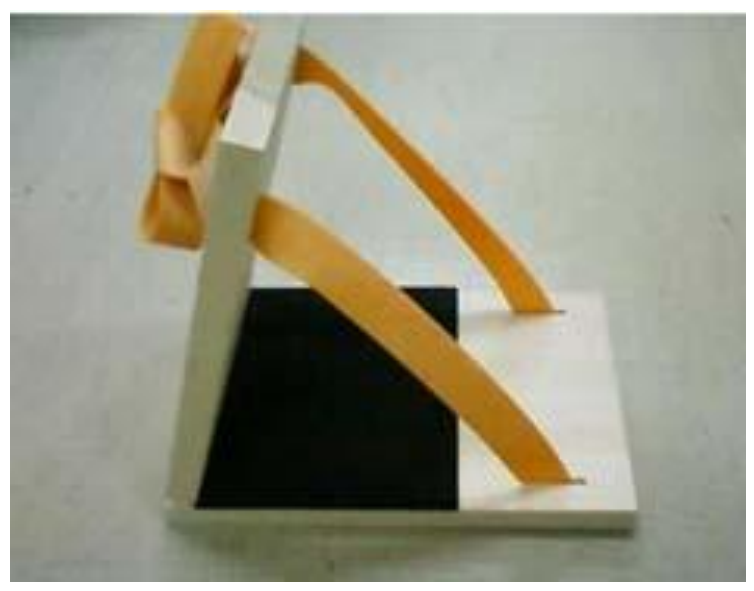

Fig. 1B. Special Pata for $\mathrm{f}$ MRI

( steel spring to rubber)

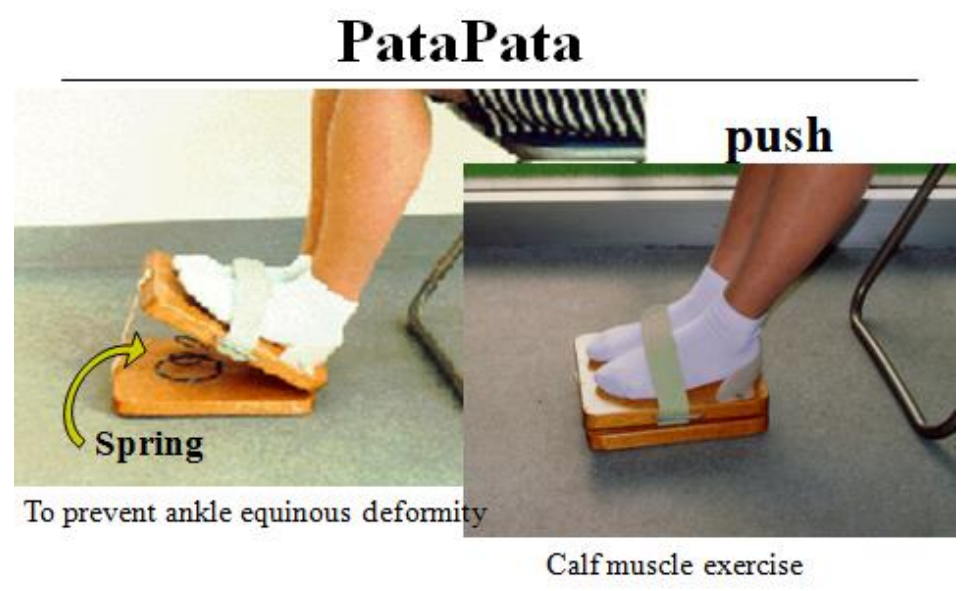

Fig. 1C. How to exercise using Pata

\subsection{How to use Pata:}

Pata is one of the tools in Motivative Exercise. We put both feet on the Pata and push it against the spring rhythmically and repeat these movements several times. Calf muscle exercises can also prevent ankle equinus deformity by working against the spring to assist stretching the calf muscle. (Figs. 1A-C) 


\section{Result}

\section{1. f MRI findings of 4 cases}

\subsubsection{The 1st case}

The 1st case is a 69 year-old-female, the left hemiplegia suffered from cerebral embolism in right MCA with a wide infarction of right temporal lobe (Fig. 2A). The onset of her illness was Dec.13, 2008 when she was 68 . The wide orange area activated with Motivative Exercise by brain function can be recognized both in the affected right parietal and non-affected left lobe (Fig. 2B). In passive ROM exercise, there is less orange area than in the Motivative Exercise (Fig. 2C).

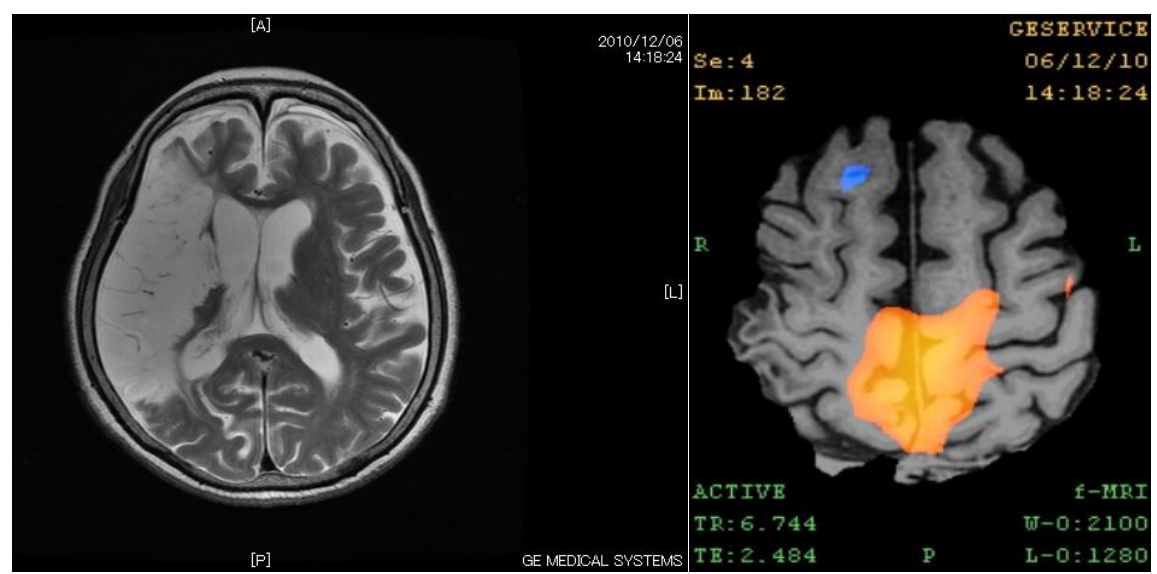

Fig. 2A. Wide right temporal infarction
Fig. 2B. Active ex.with Pata

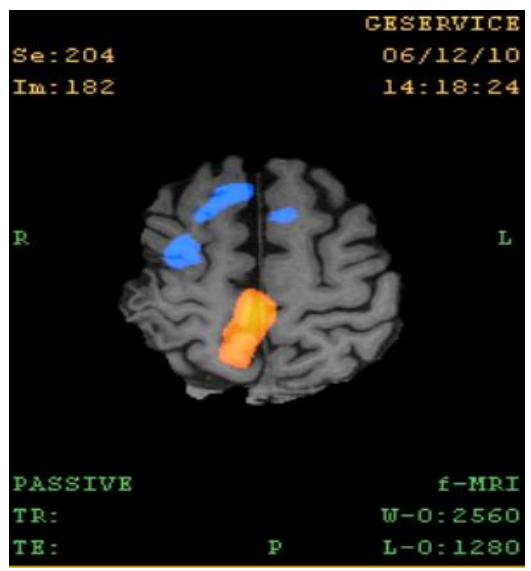

Fig. 2C. Passive ROM to left ankle joint by PT

\subsubsection{The 2 nd case}

The 2nd case is a 76-year-old female whose left hemiparesis suffered from multiple cerebral infarction (Fig. 3A). The onset of her illness was Aug. 52008 when she was 73. The orange area activated brain function in both occipital lobes can be recognized in the Motivative Exercise (Fig. 3B). In passive ROM exercise by PT, there is no orange area in either of the lobes (Fig. 3C).
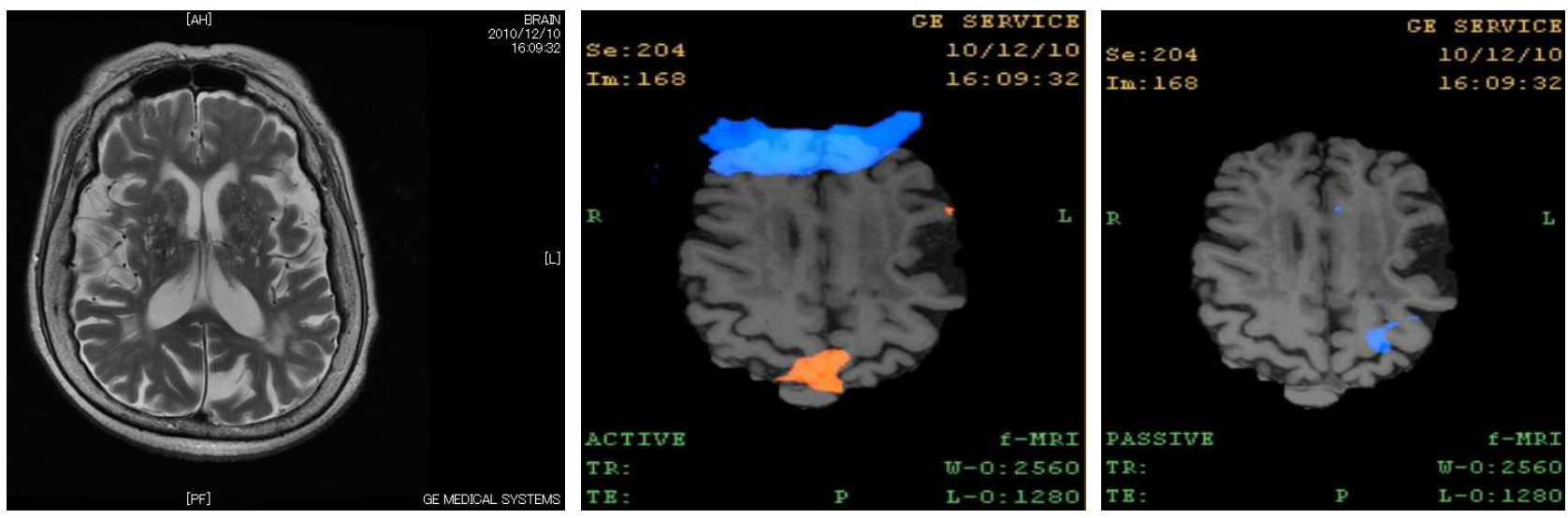

Fig. 3A. Multiple cerebral infarction

Fig. 3B. Active exercise with Pata

Fig. 3C. Passive ROM by

PT 


\subsubsection{The 3rd case}

The 3rd case is a 54-year-old male whose right hemiplegia suffered from left putaminal bleeding, post removal of hemorrhage (Fig. 4A). The onset of his illness was March 23, 2009 when he was 52. $\mathrm{He}$ is a right hemiplegic community walker. Brunnstrom stage of right LE is 2 . The orange area as activated brain function in both parietal lobes by an active Motivative Exercise with Pata (Fig. 4B) can be recognized but less in passive ROM exercise by PT (Fig. 4C).

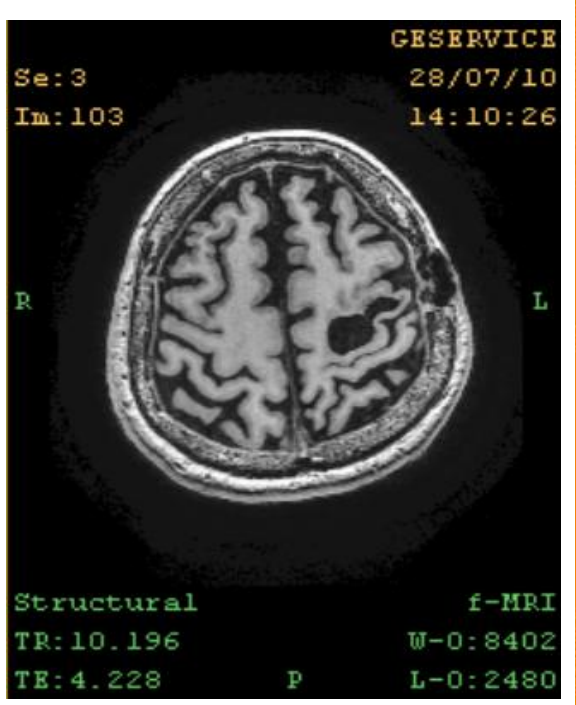

Fig. 4A. Left putaminal bleeding post-op

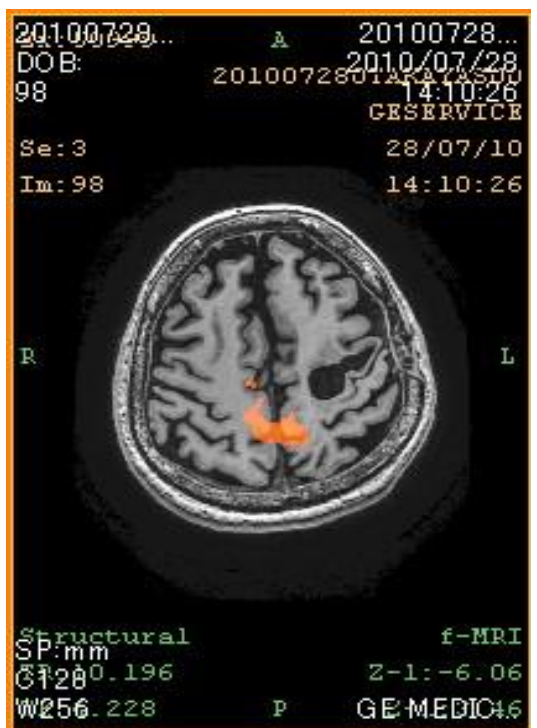

Fig. 4B. Active ex. with Pata

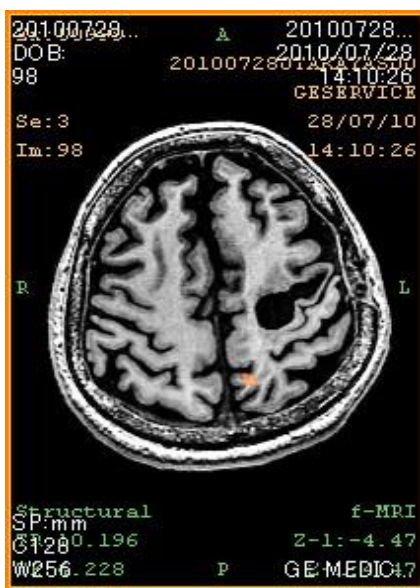

Fig. 4C. Passive ROM

\subsubsection{The 4th case}

The 4th case is 72-year-old female whose left hemiplegia suffered from right thalamic bleeding (Fig. 5A). The onset of her illness was Nov. 12, 2006 when she was 69. A wider orange area of activated brain function in not only the unaffected but also the affected right lobe can be recognized in the Motivative Exercise (Fig. 5B). Much less orange area in the right affected parietal-occipital lobe during passive ROM exercise (Fig. 5C) can be recognized.
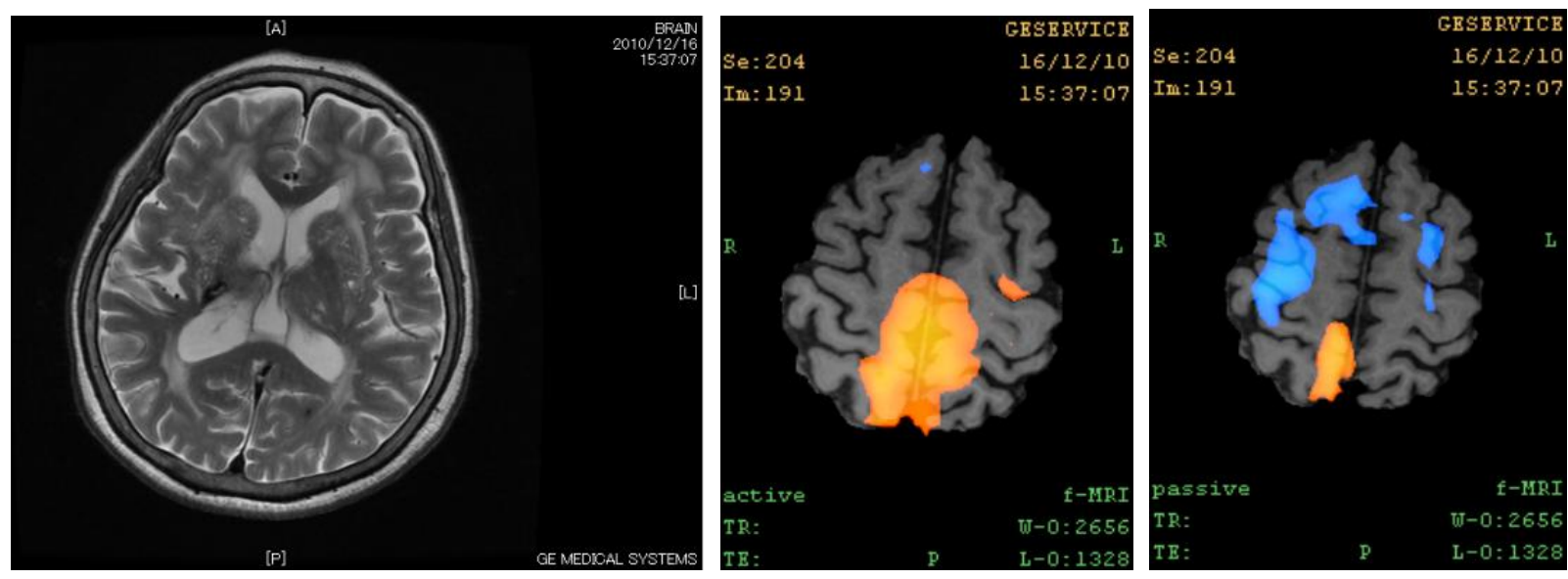

Fig. 5A. Right thalamic bleeding
Fig. 5B. Motivative Exercise Fig. 5C. Passive ROM ex. 


\section{Conclusion}

We have tried to prove the significance of Motivative Exercise compared with conventional passive ROM exercise taken by fMRI and are proving to get good results. We can imagine oxygenated, activated function in the orange areas by fMRI. The active exercise by the other side of the affected limb can definitely influence the affected side of brain. We find that the passive ROM exercise by therapist's hands can't produce brain activity and can limit the local and partial effect only.

The next question we have to think about is "Where does the orange area coincidence to central motor cortex localization?" Virtual evidence gotten by fMRI is not enough to persuade the real meanings. We must precisely study the activated orange area at depth and reasonable position fitted to the functional anatomical map.

\section{Discussion}

These results show that this simple exercise device, Pata, is useful and helpful to continue training a hemi paretic limb, and these exercises support patients abilities to rehabilitate not only ADL but also maintain their impairments independently. We are sure to expect that the Motivative Exercise is one of the most peaceful weapons against the coming aging crisis.

\section{Acknowledgement}

This work was supported by JSPS KAKENHI Grant Number 21249036.

\section{References}

1) Leighton Chan, et al "The Effect of Medicare's Payment System for Rehabilitation Hospitals on Length of Stay, Charges, and Total Payments" New England Journal of Medicine, 1997-337, pp978-985

2) Yoshiko, Morita, Aoi Fujita, Tetsuhiko Kimura, Mitsuo Makita, Hideo Kijima, Satoshi Endo, Yuzo Okamoto, Hiroshi Nagasawa, Kentaro Ngaoka, Koharu Miyamori, "The efficient Physical therapy treatment by Takizawa Method" $37^{\text {th }}$ Japanese Association of Rehabilitation Medicine, 2000/6/24.(JP)

3) Kenji Ushizawa, Takizawa Shigeo, Hiroshi Nagasawa, Mitsuyo Makita, Tetsuhiko Kimura, Kyoko Takizawa. "Statistical Evaluation of Rehabilitation based Takizawa-Program for Elderly People”, Biophilia Rehabilitation Journal (2) pp71-80. 2004

4) Yoshiko Morita, Yuzo Okamoto, Takizawa Shigeo, Kyoko Takizawa, Tetsuhiko Kimura, "Evaluation of the motivative exercise implementation based on Takizawa Rehab method",BRJ,1(1) 51-54,2002(JP)

5) TAKIZAWA, Shigeo and Kyoko Takizawa, Method for Managing Exercise for Function Recovery and Muscle Strengthening, US PAT 7153250, 2006 